\title{
The feasibility of electromagnetic navigation-guided percutaneous microcoil localization for thoracoscopic resection of small pulmonary nodules
}

\author{
Po-Kuei Hsu, MD, PhD, and Yu-Chung Wu, MD, Taipei, Taiwan
}

\footnotetext{
From the Division of Thoracic Surgery, Department of Surgery, Taipei Veterans General Hospital and School of Medicine, National Yang-Ming University, Taipei, Taiwan.

Disclosures: Authors have nothing to disclose with regard to commercial support.

Received for publication Sept 20, 2018; revisions received Nov 12, 2018; accepted for publication Nov 18, 2018 ; available ahead of print Jan 18, 2019.

Address for reprints: Po-Kuei Hsu, MD, PhD, Division of Thoracic Surgery, Department of Surgery, TaipeiVeterans General Hospital, No. 201, Section 2, Shih-Pai Rd, Taipei, Taiwan (E-mail: hsupokuei@yahoo. com.tw).

J Thorac Cardiovasc Surg 2019;157:e211-4

$0022-5223 / \$ 36.00$

Copyright (C) 2018 by The American Association for Thoracic Surgery

https://doi.org/10.1016/j.jtcvs.2018.11.101
}

Video clip is available online.

Surgical removal of small pulmonary nodules, especially nonvisible and nonpalpable ground-glass nodules, is sometimes challenging during minimally invasive thoracoscopic surgery. Preoperative nodule localization is therefore of paramount importance for precise resection. ${ }^{1,2}$ The advent of electromagnetic navigation bronchoscopy technology has led to an increasing use of electromagnetic navigation bronchoscopy-guided pleural dye marking in preoperative localization. ${ }^{3,4}$ The SPiN Thoracic Navigation System (Veran Medical Technologies, St Louis, Mo) thoracic electromagnetic navigation system combines endobronchial and percutaneous navigation. ${ }^{5}$ A Chiba needle with a stylet that contains a sensor is used for electromagnetic navigation. For peripheral nodules that lie outside the airway and are difficult to access by bronchoscopy, a transthoracic approach becomes another choice. This novel technology has been used to increase the diagnostic yield of pulmonary nodules. ${ }^{6}$ There have been no reports about electromagnetic navigation-guided percutaneous microcoil localization for thoracoscopic resection of small pulmonary nodules, however, so we aimed to investigate the feasibility of this technique in facilitating target nodule identification during minimally invasive thoracoscopic surgery.

\section{TECHNIQUES}

This study was reviewed by the institutional review board of the Taipei-Veterans General Hospital. Electromagnetic navigation was performed with the SPiN Perc Thoracic Navigation System (Veran Medical Technologies), which

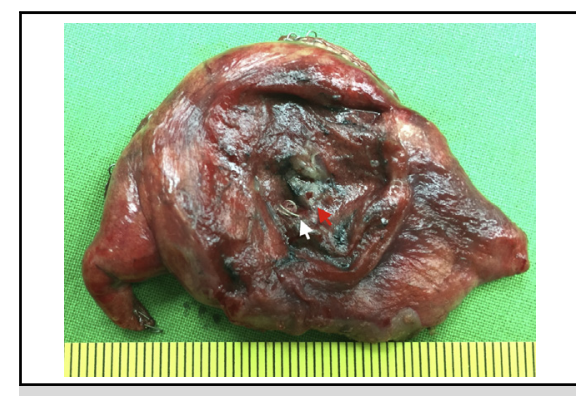

Precise localization with the microcoil very close to the target nodule.

\section{Central Message}

Electromagnetic navigation-guided percutaneous microcoil localization could facilitate identification of small pulmonary nodule during thoracoscopic surgery.

See Commentaries on pages e 215 and e217.

required a baseline inspiration and expiration computed tomographic scan after placement of a navigational tracking pad on the patient's chest. DICOM-formatted images were transferred to the planning software for the selection of a chest wall entry point and navigational pathway to the target lesion. During the localization procedure, electromagnetic navigation allowed real-time tracking of the 19-gauge Chiba needle by detecting the position of the electromagnetic tip-tracked needle stylet (ETTNS). The needle was placed on top of the planned entry point, and the angle to the target lesion was selected with the electromagnetic navigation system guidance (Figure 1, $A$ and $B$, and Video 1). The needle was then advanced through the chest wall toward the target lesion. When the target lesion was reached, the ETTNS was removed, and a microcoil (MicroNester Coil; Cook Medical, Bloomington, Ind) was inserted through the 19-gauge needle and deployed into the lung parenchyma. The ETTNS was inserted again to confirm the needle location. For dual localization with dye and microcoil, the needle was withdrawn to about $0.5 \mathrm{~cm}$ from the visceral pleura, the ETTNS was removed again, and $0.2 \mathrm{~mL}$ of dye (Patent Blue V 2.5\%; Guerbet, Aulnay-sous-Bois, France) was injected to complete the localization procedure. For dual localization with 


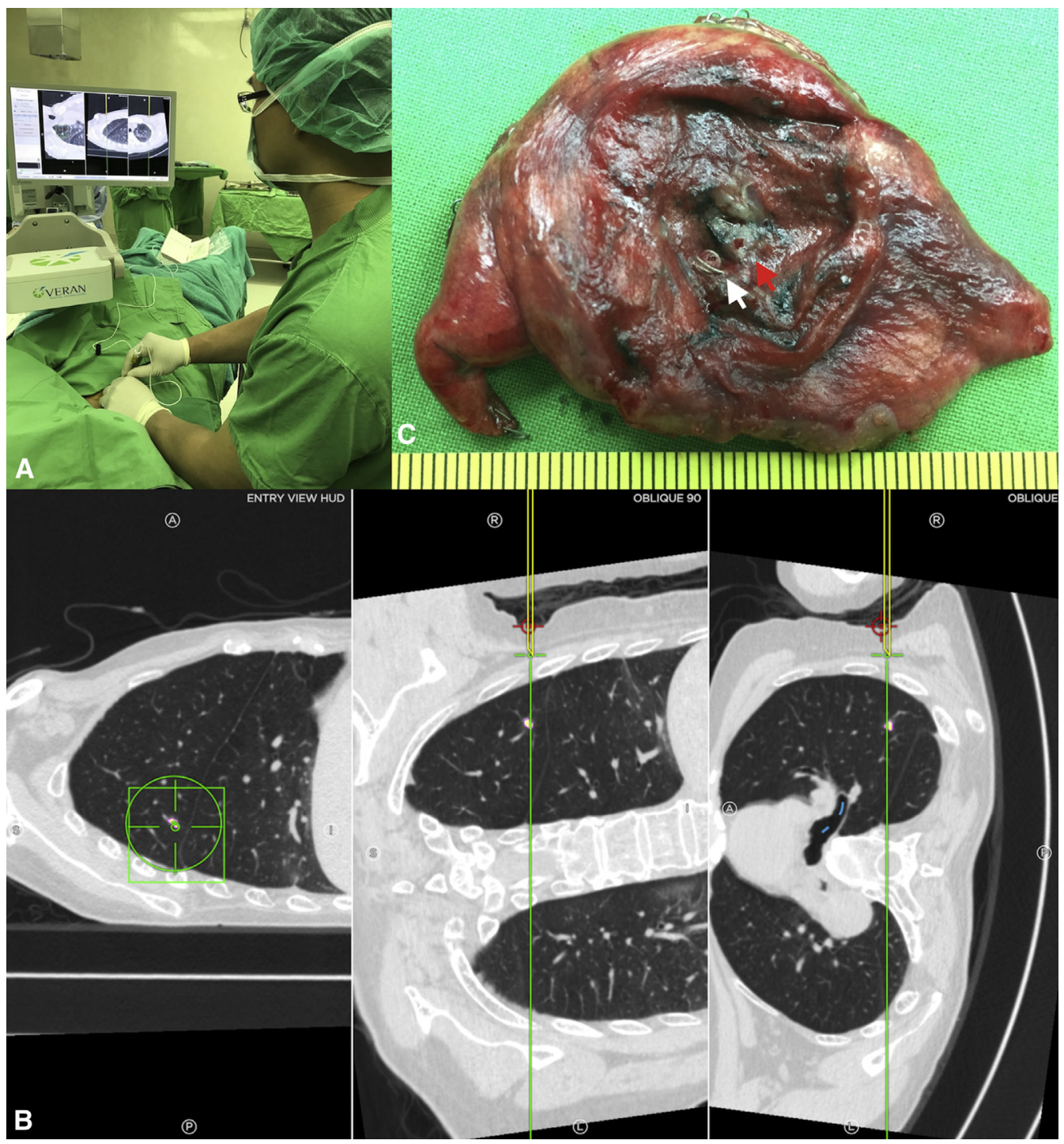

FIGURE 1. A, Electromagnetic navigation-guided localization was based on observation of the navigation system screens. The needle was placed on top of the planned entry point, and the angle to the target lesion was selected with the navigation system guidance. B, The entrance point (red circle) and the path (green) to the target were shown in transverse and coronal views on the screen. While the needle was advancing through the chest wall toward the target lesion, electromagnetic navigation allowed real-time tracking of the electromagnetic tip-tracked needle stylet (yellow). C, After resection, the shortest distance between the nodule and microcoil was measured. In this case, localization was precise, with the microcoil (white arrow) very close to the target nodule (red arrow).

indocyanine green and microcoil, $0.3 \mathrm{~mL}$ of indocyanine green $(0.125 \mathrm{mg} / \mathrm{mL})$ was injected. In patients with a dual-localization procedure, the position of the target lesion was identified either by the blue dye over the visceral pleural surface or with the guidance of a near-infrared fluorescence imaging system (PINPOINT Endoscopic Fluorescence Imaging System; Novadaq Technologies Inc, Mississauga, Ontario, Canada). All localization procedures were done with the patient under general anesthesia in the operating room and followed immediately by thoracoscopy. The lung targeted for resection was clamped by thoracoscopic instruments, and adequate distance between the microcoil and instruments was confirmed by C-arm fluoroscopy before application of the endostaplers for resection.

After resection, the exact positions of the pulmonary nodule and microcoil were confirmed by palpation before the specimen was cut. The shortest distance between the nodule and microcoil was measured to evaluate the accuracy of the localization procedure (Figure 1, C). This technique was successfully used in 6 patients between May 2018 and August 2018. Table 1 shows the patient characteristics. It must be emphasized that the distance between the nodule and microcoil was less than $0.5 \mathrm{~cm}$ in 4 


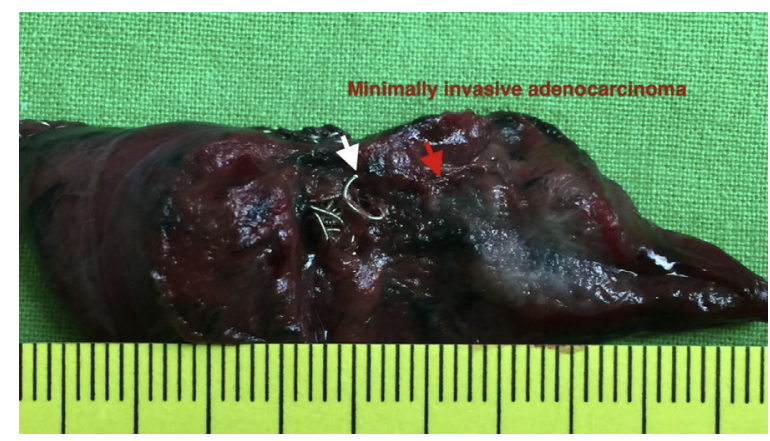

VIDEO 1. This video describes the electromagnetic navigation-guided percutaneous microcoil localization for thoracoscopic resection of small pulmonary nodules. This novel system uses a Chiba needle with a stylet that contains a sensor. The electromagnetic navigation tracks the needle by detecting the position of the electromagnetic tip-tracked needle stylet in real time. When the needle reaches the target nodule, the stylet is removed, and a microcoil, indocyanine green, or blue dye injection can be used to mark the lesion. The procedure relies on observation of the electromagnetic navigation system screens. The needle entry point and the angle to the target lesion is selected with the electromagnetic navigation system's guidance. The first case is that of a patient with a ground-glass opacity $(G G O)$ lesion (arrow) of the right upper lobe $(R U L)$. The green line represents the trajectory to the target nodule. While the needle was advancing through the chest wall toward the target lesion, electromagnetic navigation allowed real-time tracking of the electromagnetic tip-tracked needle stylet, which is shown in yellow on the screen. When the target nodule was reached, the stylet was removed and a microcoil was deployed into the lung parenchyma. During the thoracoscopic procedure, the lung targeted for resection was clamped, and the position of the microcoil (yellow arrow) was confirmed with intraoperative fluoroscopy. In the resected specimen, the coil, indicated by the white arrow, was very close to the target nodule, which is indicated by the red arrow. The second case is that of another patient with a ground-glass opacity $(G G O)$ lesion (red arrow) of the right upper lobe $(R U L)$. The needle was navigated toward the target nodule in a similar fashion to that in the first case. In addition to a microcoil, indocyanine green was also used for marking in this case and the position of the target nodule was clearly identified during the thoracoscopic procedure. In the resected specimen, the coil, indicated by the white arrow, was very close to the target nodule, which is indicated by the red arrow. The third case is that of a patient with a ground-glass opacity $(G G O)$ lesion (arrow) of the left lower lobe ( $L L L)$. Because we planned to approach the nodule from the dorsal side, we obtained a decubitus computed tomographic scan, which was in the same position during the localization procedure. The software indicated the optimal skin entry point, and electromagnetic navigation allowed real-time tracking of the electromagnetic tip-tracked needle stylet. We intended to advance the needle further and deploy the microcoil deeper to ensure an adequate resection margin. In this case, blue dye was used to mark the nodule position on the pleural surface. In the resected specimen, the coil, indicated by the white arrow, is very close to the target nodule, which is indicated by the red arrow. Video available at: https://www.jtcvs.org/article/S0022-5223(18)33251-3/fulltext.

cases, between 0.5 and $1 \mathrm{~cm}$ in 1 case, and greater than $1 \mathrm{~cm}$ in 1 case, with an average distance of $4.2 \pm$ $5.0 \mathrm{~mm}$. There were no complications such as bleeding or embolism.
TABLE 1. Patient characteristics

\begin{tabular}{|c|c|}
\hline Age $(y ;$ mean \pm SD) & $59.5 \pm 15.5$ \\
\hline \multicolumn{2}{|l|}{ Sex } \\
\hline Male & 2 \\
\hline Female & 4 \\
\hline \multicolumn{2}{|l|}{ Location } \\
\hline Right upper lobe & 3 \\
\hline Right middle lobe & 1 \\
\hline Left upper lobe & 1 \\
\hline Left lower love & 1 \\
\hline Nodular size $(\mathrm{cm}$; mean $\pm \mathrm{SD})$ & $0.75 \pm 0.18$ \\
\hline \multicolumn{2}{|l|}{ Nodular characteristics } \\
\hline Solid & 2 \\
\hline Partially solid & 2 \\
\hline Pure GGO & 2 \\
\hline $\begin{array}{l}\text { Distance between pleura and nodule } \\
\qquad(\mathrm{mm} ; \text { mean } \pm \mathrm{SD})\end{array}$ & $8.5 \pm 6.8$ \\
\hline $\begin{array}{l}\text { Target motion between inspiration and expiration } \\
\quad(\mathrm{mm} ; \text { mean } \pm \mathrm{SD})^{*}\end{array}$ & $14.6 \pm 12.4$ \\
\hline $\begin{array}{l}\text { Distance between chest wall entry site and nodule } \\
\quad(\mathrm{mm} ; \text { mean } \pm \mathrm{SD})^{*}\end{array}$ & $60.4 \pm 31.4$ \\
\hline Localization procedure time (min; mean $\pm \mathrm{SD}$ ) & $11.7 \pm 4.9$ \\
\hline \multicolumn{2}{|l|}{ Localization method } \\
\hline Microcoil & 1 \\
\hline Microcoil + dye & 3 \\
\hline Microcoil + ICG & 2 \\
\hline $\begin{array}{l}\text { Distance between microcoil and nodule } \\
\qquad(\mathrm{mm} ; \text { mean } \pm \mathrm{SD})\end{array}$ & $4.2 \pm 5.0$ \\
\hline $\begin{array}{l}\text { Distance between staple margin and nodule } \\
\qquad(\mathrm{mm} \text {; mean } \pm \mathrm{SD})\end{array}$ & $10.5 \pm 4.6$ \\
\hline \multicolumn{2}{|l|}{ Diagnosis } \\
\hline Minimally invasive adenocarcinoma & 4 \\
\hline Metastasis & 2 \\
\hline
\end{tabular}

Data are number of patients unless otherwise noted. $S D$, Standard deviation; $G G O$ ground-glass opacity; $I C G$, indocyanine green. *Information provided by the navigation software.

\section{DISCUSSION}

Electromagnetic navigation can determine the spatial location of a sensor device within a magnetic field. By combining this spatial information with previously obtained computed tomographic images, the system can display the sensor device's position in relation to the anatomy. ${ }^{3}$ The novel electromagnetic navigation system even includes miniature tracking sensors built into biopsy needles to allow real-time tracking during percutaneous procedures. Another feature of this novel system is respiratory gating technology that can track nodule movement during patient breathing throughout the procedure. Ideally, this eliminates the need for fluoroscopy or cone-beam computed tomographic confirmation. In this report, we proposed dual localization, combining the advantages of dye and indocyanine green fluorescence, which identify the position 
of the target lesion on the pleural surface, with microcoil placement, in which the distance between the microcoil and the resection could be confirmed by intraoperative fluoroscopy. This is the first report to demonstrate the feasibility and accuracy of electromagnetic navigation-guided percutaneous preoperative localization for small pulmonary nodules, facilitating target nodule identification during thoracoscopic surgery. More case experience is needed to confirm our observations in the future.

\section{References}

1. Lin MW, Tseng YH, Lee YF, Hsieh MS, Ko WC, Chen JY, et al. Computed tomography-guided patent blue vital dye localization of pulmonary nodules in uniportal thoracoscopy. J Thorac Cardiovasc Surg. 2016;152:535-44.e2.
2. Hsieh MJ, Wen CT, Fang HY, Wen YW, Lin CC, Chao YK. Learning curve of image-guided video-assisted thoracoscopic surgery for small pulmonary nodules: a prospective analysis of 30 initial patients. J Thorac Cardiovasc Surg. 2018;155: 1825-32.e1.

3. Mehta AC, Hood KL, Schwarz Y, Solomon SB. The evolutional history of electromagnetic navigation bronchoscopy: state of the art. Chest. 2018;154: 935-47.

4. Abbas A, Kadakia S, Ambur V, Muro K, Kaiser L. Intraoperative electromagnetic navigational bronchoscopic localization of small, deep, or subsolid pulmonary nodules. J Thorac Cardiovasc Surg. 2017;153:1581-90.

5. Arias S, Lee H, Semaan R, Frimpong B, Ortiz R, Feller-Kopman D, et al. Use of electromagnetic navigational transthoracic needle aspiration (E-TTNA) for sampling of lung nodules. J Vis Exp. 2015;99:e52723.

6. Thiboutot J, Lee HJ, Silvestri GA, Chen A, Wahidi MM, Gilbert CR, et al. Study design and rationale: a multicenter, prospective trial of electromagnetic bronchoscopic and electromagnetic transthoracic navigational approaches for the biopsy of peripheral pulmonary nodules (ALL IN ONE Trial). Contemp Clin Trials. 2018;71:88-95. 